\title{
Foreword: Earth's gravity field and Earth sciences
}

\author{
Fernando Sansò ${ }^{1} \cdot$ Riccardo Barzaghi $^{2}$
}

Received: 12 September 2020 / Accepted: 15 September 2020 / Published online: 10 October 2020

(c) Accademia Nazionale dei Lincei 2020

\section{Graphic abstract}

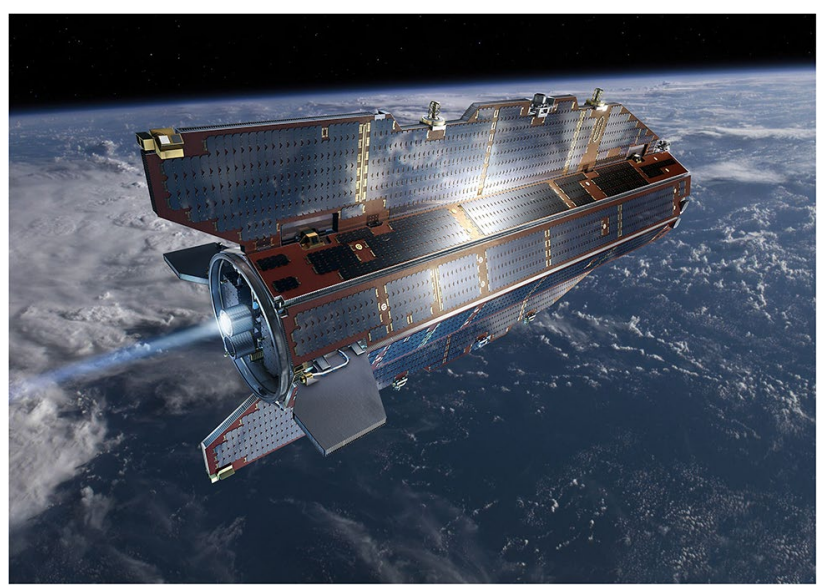

The gravity field of the Earth is a complicated object; it contains useful information spread over nine orders of magnitude, from $\mathrm{g}\left(10^{3} \mathrm{Gal}\right)$ to $10^{-6} \mathrm{~g}(1 \mu \mathrm{Gal})$. The whole range is observable but basically with very different tools: from the very long wavelength components, of the order of the radius of the Earth, that we can best estimate observing the orbits of high satellites, to the medium range, down to $100 \mathrm{~km}$, that are observed both from the space (GRACE and GOCE) and from ground by ordinary gravimetry - and also, for most areas of the surface of the planet, from the inversion of radar-altimetry on the oceans, to the short range, down to $1 \mathrm{~km}$ (which at present can be achieved only by ground or aerial observations). Finally the measurements performed at isolated permanent stations, nowadays by cold atoms interferometry, can achieve the accuracy of $1 \mu \mathrm{Gal}$ that have the

Riccardo Barzaghi

riccardo.barzaghi@polimi.it

Fernando Sansò

fernando.sanso@polimi.it

Accademia Nazionale dei Lincei, Rome, Italy

2 Politecnico di Milano, Milan, Italy function of benchmarks for other gravity observations and accurately display the time variations of the field.

As one recognizes from this fast ride through the measurements relative to the gravity field, a very large number of physical phenomena are used for the observations: bringing back all this information to a unitary vision of the field is a gigantic effort from the theoretical, the numerical as well as the informatic points of view. From the start, the geodetic community working on gravity field determination has to be able to interact with astronomers (for the interplay with orbit tracking), with physicists (to model the observation equations), with geologists/geophysicists (to understand features of crustal dynamics and their information on prior gravity values), with oceanographers (to understand the interplay between geoid and ocean dynamics which are both contained in the altimetry measurements) and with "Earth observers" (providing an indispensable information on the shape of the topographic shell). With no claim of foreseeing the far future, we can comment that present achievements can only be seen as a step in a process along a path with highly challenging obstacles. 
Of course, the International Association of Geodesy, in cooperation with sister Associations and other institutions, keep constant track and stimulate this process. Among them, we would like to include the Accademia dei Lincei, that, though national by its very nature, due to its old militancy on the battlefield of science (in fact the oldest in the world), has agreed to organize this conference in which we try to make the point on where we are in the knowledge of the gravity field at the international and national level. It is not necessary to go back to Galileo (Lincei academician from 1611), let us just mention others famous academicians that contributed to the understanding of the gravity field, such as Paolo Pizzetti (1860-1918), Linceo from 1903, Carlo Somigliana (1860-1955), Linceo from 1898, Antonio Marussi (1908-1984), Linceo from 1961; to Michele Caputo, Linceo from 1970, to whom articles in the previous Special Issue in this series have been dedicated. Inspired by their work, periodically we attempt at organizing in the Accademia a conference covering different branches of Geodesy.

We had a conference in 2014 on Geodesy and Geomatics (Sansò and Crespi 2015) (Fig. 1), and a second one in 2017 on Satellite Positioning (Crespi et al. 2018) (Fig. 2). The articles in this issue collects the third conference on the theme of Gravity. As in the previous occasions, we have

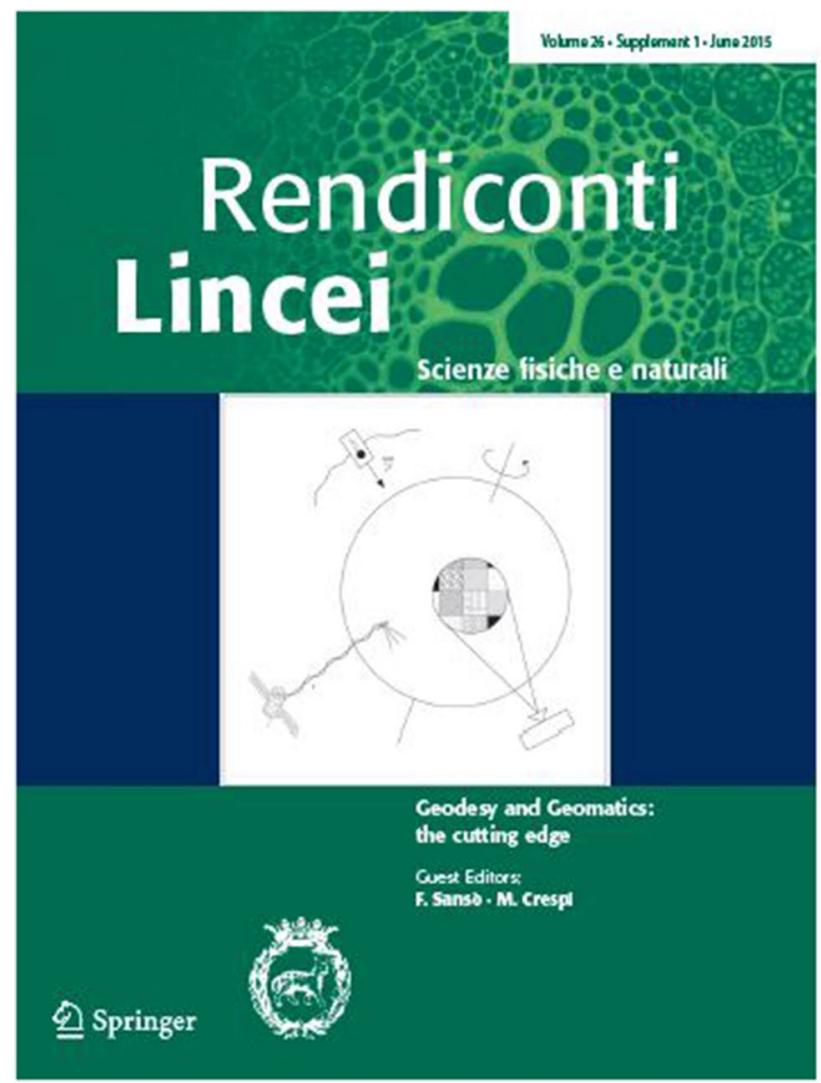

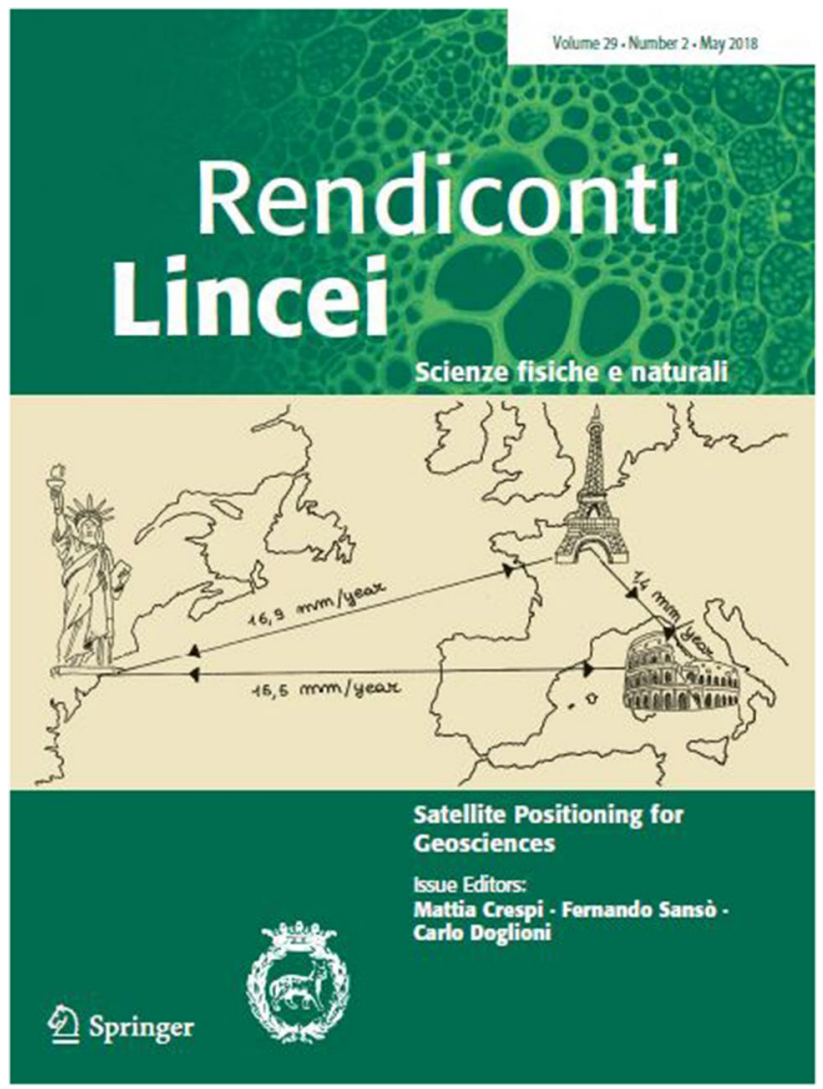

Fig. 2 Cover of Crespi et al. 2018

been guided by interdisciplinarity, to the aim of establishing connections among Geodesy and other disciplines continuing and enhancing fruitful cooperation.

\section{References}

Sansò F, Crespi M (eds) (2015) Geodesy and geomatics: the cutting edge. 26(Supplement 1):S1-S125

Crespi M, Sansò F, Doglioni C (eds) (2018) Satellite positioning for geosciences. 29(Supplement 1):S1-S108

Publisher's Note Springer Nature remains neutral with regard to jurisdictional claims in published maps and institutional affiliations.

Fig. 1 Cover of Sansò and Crespi 2015 